\title{
Caractérisation Hydrogéochimique des Aquifères du Bassin de Timia (Massif de l'Aïr, Nord du Niger)
}

\author{
Illias Alhassane, PhD \\ Université d'Agadez Faculté des Sciences et Techniques, \\ Département de Géologie, Agadez, Niger \\ Abdou Babaye Maman Sani, M.A. \\ Université Dan Dicko Dankoulodo Faculté des Sciences et Techniques, \\ UMR SERMUG, Département de Géologie, Maradi, Niger \\ Sandao Issoufou, M.A. \\ Université Abdou Moumouni de Niamey Faculté des Sciences et \\ Techniques, Département de Géologie, Niamey, Niger \\ Issa M. Salmanou Souleymane, PhD \\ Université de Zinder Faculté des Sciences et Techniques, \\ Département de Géologie, Zinder, Niger \\ Ousmane Boureima, Pr. \\ Université Abdou Moumouni de Niamey Faculté des Sciences et \\ Techniques, Département de Géologie, Niamey, Niger
}

Doi:10.19044/esj.2019.v15n30p301 URL:http://dx.doi.org/10.19044/esj.2019.v15n30p301

\section{Résumé}

Le bassin de Timia est situé dans le massif de l'Aür (Nord du Niger). Dans cette zone, les ressources en eaux sont représentées par les aquifères altérés et fissurés du socle et ceux des alluvions. Ces aquifères, sont sollicités pour l'alimentation des populations, et pour l'irrigation. Ces activités, ont eu comme conséquence la dégradation de la qualité de ces eaux. L'objectif de la présente étude est de déterminer les caractéristiques physico-chimiques et les mécanismes d'acquisition de la minéralisation des eaux souterraines de la zone de Timia. La méthodologie basée sur les approches hydrochimiques et l'analyse en composante principale montre que les facies chimiques des eaux souterraines sont dominés par les facies de type bicarbonatés calciques et magnésiens (52\%). Les facies bicarbonatés sodiques et potassiques sont aussi important (29\%) tandis que les eaux hyper-bicarbonatées sodiques (13\%) sont faiblement représentées. Les sources de la minéralisation des eaux sont principalement de trois types : les apports superficiels (pollution), l'hydrolyse des minéraux silicatés et les activités volcaniques. 
Mots clés: Aquifères fissurés, Nappe alluviale, Hydrochimie, Minéralisation, Niger

\title{
Hydrogeochemical Characterization of Timia Basin Aquifers (Aïr massif, Northern Niger)
}

\author{
Illias Alhassane, PhD \\ Université d'Agadez Faculté des Sciences et Techniques, \\ Département de Géologie, Agadez, Niger \\ Abdou Babaye Maman Sani, M.A. \\ Université Dan Dicko Dankoulodo Faculté des Sciences et Techniques, \\ UMR SERMUG, Département de Géologie, Maradi, Niger \\ Sandao Issoufou, M.A. \\ Université Abdou Moumouni de Niamey Faculté des Sciences et \\ Techniques, Département de Géologie, Niamey, Niger \\ Issa M. Salmanou Souleymane, PhD \\ Université de Zinder Faculté des Sciences et Techniques, \\ Département de Géologie, Zinder, Niger \\ Ousmane Boureima, Pr. \\ Université Abdou Moumouni de Niamey Faculté des Sciences et \\ Techniques, Département de Géologie, Niamey, Niger
}

\begin{abstract}
The Timia Basin is located in the Aïr Massif (Northern Niger). In this area, water resources are represented by altered and fissured basement and alluvial aquifers. These aquifers, are solicited for the water supply, and irrigation. These activities have for consequences the degradation of the quality of these waters. The objective of this study is to determine the physicochemical characteristics and the mechanisms house control the mineralization of groundwater in Timia area. The methodology based on hydrochemical approaches and principal component analysis shows that groundwater chemistry are dominated by calcium-magnesium bicarbonate facies (52\%). Sodium-potassium bicarbonate facies are also important (29\%) while, sodium hyper-bicarbonate waters $(13 \%)$ are poorly represented. The sources who controlled the water mineralization are mainly of three types: superficial supply (pollution), hydrolysis of silicate minerals and volcanic activities.
\end{abstract}


Keywords: Fractured aquifers, Alluvium, Hydrochemistry, Mineralization, Niger

\section{Introduction}

Depuis 1970, l'Afrique de l'Ouest a connu une baisse drastique des précipitations en particulier dans le sahel (Lebel and Ali, 2009). Dans la partie nord du Niger, en particulier dans l'Aïr, où se situe la zone d'étude, le besoin en eau de la population et du cheptel est assuré par les nappes alluviales peu profondes. Ces dernières sont utilisées pour l'irrigation et les cultures maraîchères (agrumes, céréales, pommes de terre, oignons, etc...). L'utilisation abusive des engrais chimiques et des pesticides, a eue pour conséquence la dégradation de la qualité des eaux de ces nappes superficielles, nécessitant l'exploitation des aquifères plus profonds de socle altéré et ou fissuré dont certains points montrent d'importante teneurs en fluor dépassant les normes des qualités définies par l'OMS (Illias, 2018). Ainsi, l'évolution et le suivie de la qualité physico-chimique de ces eaux souterraines du massif de l'Aïr s'avèrent indispensables en vue d'une gestion intégrée des dites ressources. Les études hydrochimiques des eaux du massif de l'Aïr remontent aux travaux de (Garnier et Fontes, 1980 ; Roussin-Bariac, 1985 ; Aranyossy et Joseph, 1985 ; Joseph et Frangi, 1989 ; Joseph, 1990 ; Gallaire, 1992 ; Illias, 2018). Depuis ces travaux, les aquifères de cette localité n'ont été l'objet d'aucune étude portant sur leurs caractéristiques hydrochimiques. C'est dans cette perspective que s'inscrit la présente étude qui a pour objectif principal de déterminer les caractéristiques hydrochimiques des eaux des aquifères de la zone de Timia et de déterminer les sources et les facteurs qui contrôlent leur minéralisation.

\section{Généralités sur la Zone d'étude}

Le bassin versant de Timia (Figure 1), est situé dans la partie centrale de l'Aïr entre $17^{\circ} 50^{\prime}$ et $18^{\circ} 15^{\prime}$ de latitudes Nord et $8^{\circ} 30^{\prime}$ et $9^{\circ} 00^{\prime}$ de longitudes Est. Avec une superficie d'environ $267,5 \mathrm{~km}^{2}$ (Gallaire, 1995). Le climat de la zone de Timia, est du type sahélien. Les hauteurs pluviométriques enregistrées à la station de Timia en 2009 et en 2008 sont respectivement de $101,58 \mathrm{~mm}$ et de 117,02 mm (cellule COGERAT, 2008). Sagissant des températures, les plus basses $\left(11,5^{\circ} \mathrm{C}\right)$ sont observées en décembre-février (saison froide). Par contre, les fortes valeurs de températures $\left(41,2{ }^{\circ} \mathrm{C}\right)$ sont enregistrées en avril-juin (saison sèche). La végétation se rencontre dans les vallées et sur certaines plaines inondables (Schulz, 1987). 

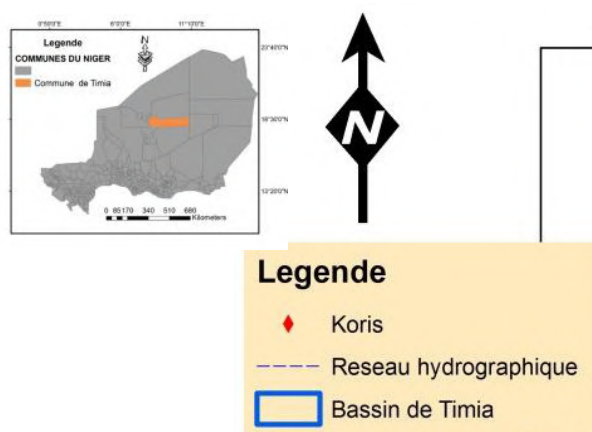

Bassin versant de Timia Altitudes (m)
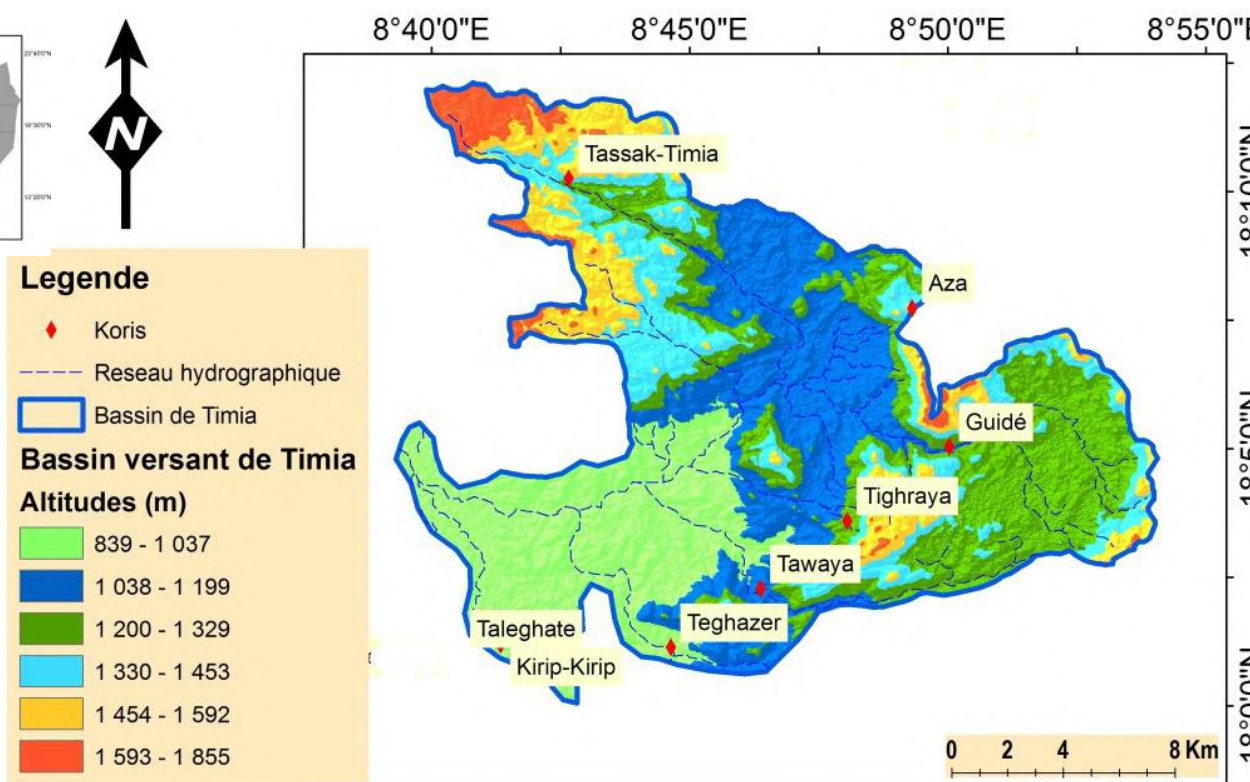

Figure 6: Présentation de la zone d'étude et le réseau hydrographique

\section{Contexte géologique et hydrogéologique}

D'après les travaux de (Raulais, 1959; Greigert et al., 1967 ; Black, 1967 ; Moreau, 1982), quarte grandes unités caractérisent le massif de l'Aïr : (i) le Socle représenté par un ensemble métamorphique présentant un bombement dissymétrique légèrement déversé vers l'Est (Black, et al., 1985), composé des formations gneissiques ou schisteuses (Black, 1967). (ii) les complexes subvolcaniques, au nombre d'une vingtaine (Raulais, 1959), ces structures recoupent le socle, selon les termes de Moreau (1982) a " l'emporte-pièce", sous forme des complexes à structures annulaires et résultent de l'intrusion de roches alcalines et hyperalcalines (Black et al., 1967; Karche et Vachette, 1978). (iii) le volcanisme récent dû aux mouvements du socle au Niger se traduisant dans l'Aïr par l'effondrement de Téfidet, et par l'émission, à sa bordure N-O, à la faveur du rejeu des failles préexistantes NO-SE (More1, 1985; Karche, 1979. (iiii) les formations sédimentaires, qui se rencontrent sur sa bordure Ouest dans bassin de Tim Mersoï (Valsardieu, 1971 ; El Hamet, 1983 ; Yahaya, 1992 ; Semperé, 1981). L'hydrogéologie des aquifères profonds du socle de la zone d'étude est peu connue, compte tenu, du fait que les premiers forages (17 forages dont 7 productifs) réalisés dans l'Aïr, datent seulement de 2012. Les principaux aquifères identifiés sont de bas en haut : (i) les aquifères fissurés du socle contenus dans du granite et du gneiss fissuré, leur débit varie de 1 à $8 \mathrm{~m}^{3} / \mathrm{h}$, les profondeurs des venues d'eau se situent entre 16 et $24 \mathrm{~m}$, tandis que les niveaux statiques sont compris entre 9 et $25 \mathrm{~m}$. Ces eaux sont très chargées en sels minéraux. Ce réservoir se caractérise par une porosité et une 
transmissivité faibles (Armand, 1987).Toutefois, une vingtaine de sources ont été inventoriées (Joseph, 1991). (ii) les aquifères des altérites sont localisés dans les zones altérés du socle, ils sont captés essentiellement par des puits cimentés à grand diamètre et leur profondeur moyenne est de $22 \mathrm{~m}$ environ. Les eaux sont d'une salinité moyenne, pour la plupart des ouvrages. (iii) les aquifères d'alluvions localisés sur les bords, et au fond des koris dans les formations argilo-sableuses des alluvions d'oueds constituent un réservoir important du fait de sa capacité d'emmagasinement (Joseph et Diluca, 1986), il est toutefois réduit en volume (Joseph, 1991). Ces aquifères sont captés par des puits de faible profondeur (6 à $18 \mathrm{~m}$ ). Les eaux de ces nappes ont une faible salinité.

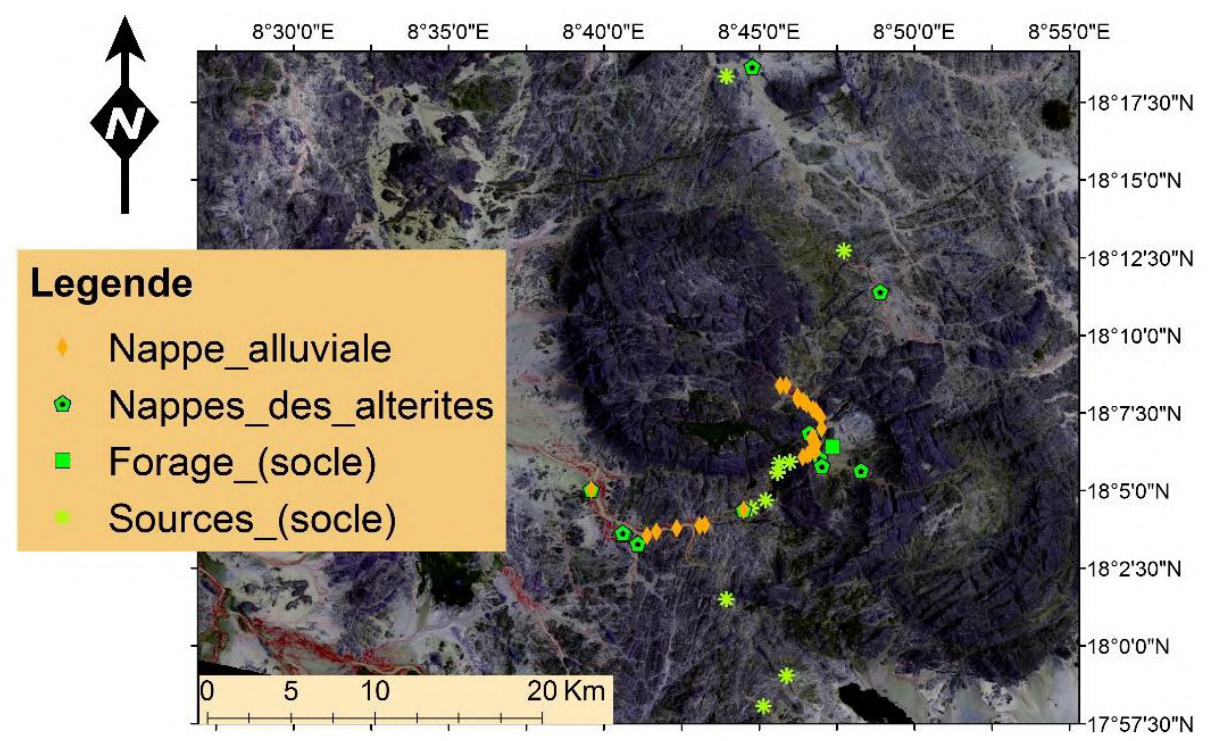

Figure 2: localisation des points échantillonnés dans la zone de Timia).

\section{Matériel et Méthodes}

\section{Echantillonnage et analyses chimiques}

Deux (2) campagnes d'échantillonnage ont été effectuées au cours de cette étude, la première réalisée en mai 2016 (période de basses eaux) au cours de laquelle 40 points d'eau ont été échantillonnés dont : 9 sources et 1 forages (aquifère du socle), 13 puits (nappe d'altérites) et 17 puits (nappe alluviale). La deuxième campagne a eu lieu en janvier 2017 (période de hautes eaux) qui a permis de prélever également 40 échantillons d'eau, dont seulement 28 sont identiques à ceux de la première campagne. Tandis que, les douze (12) autres échantillons sont des nouveaux points composés de (9 puits et 3 sources).

Les mesures in situ portant sur les paramètres physiques (conductivité, température, $\mathrm{pH}$ ) ainsi que les prélèvements ont été effectuées sur les différents ouvrages après $30 \mathrm{mn}$ de puisages afin d'avoir une eau représentative de la nappe. Ensuite, les flacons ont été rincés avec les eaux à 
prélever, puis, ils ont été remplis, étiquetés et conservés dans une glacière, pour être transportés au laboratoire d'analyses d'eau.

Les cations, dont le $\mathrm{Ca}^{2+}, \mathrm{Mg}^{2+}, \mathrm{Na}+$ et $\mathrm{K}^{+}$, ont été déterminés par absorption atomique à partir d'un spectromètre de type VARIAN-AA240FS Fast Sequential Absorption Spectrometer. Quant aux anions, dont les Chlorures $\left(\mathrm{Cl}^{-}\right)$, les Nitrates $\left(\mathrm{NO}^{-}\right)$, les Sulfates $\left(\mathrm{SO}^{--}\right)$, les Fluor $\left(\mathrm{F}^{-}\right)$, ils ont été déterminés par spectrophotométrie avec un spectrophotomètre DR 2900. Enfin, les ions bicarbonates $\left(\mathrm{HCO}_{3}{ }^{-}\right)$, ont été obtenus par abstraction à partir du TAC (titre alcalimétrique complet déterminé par titrimétrie) et du pH.

\section{Traitements des données}

Les résultats issus des différentes techniques analytiques ont été traités principalement par des techniques statistiques et des digrammes. Les différents faciès chimiques des eaux souterraines ont été déterminés à l'aide du diagramme de Piper (1944).

Pour mettre en évidence les relations entre les variables (paramètres chimiques) et regrouper celles qui ont de comportements semblables, l'analyse en composante principale (ACP) a été utilisée. Cette dernière, a été réalisée sur les 9 éléments chimiques dosés $\left(\mathrm{Mg}^{2+}, \mathrm{Ca}^{2+}, \mathrm{Na}^{+}, \mathrm{K}^{+}, \mathrm{HCO}_{3}{ }^{-}, \mathrm{Cl}^{-}, \mathrm{NO}_{3}^{-}\right.$, $\left.\mathrm{SO}_{4}{ }^{2-}, \mathrm{F}^{-}\right)$, ou déterminés $\left(\mathrm{MT}, \mathrm{pCO}_{2}\right)$ et les paramètres mesurés sur le terrain $(\mathrm{pH}, \mathrm{CE})$. De plus, les rapports ioniques ont été utilisés afin de déterminer les processus et les sources de la minéralisation des eaux souterraines.

\section{Résultats}

Les résultats des analyses des paramètres physico-chimiques sont donnés dans le tableau 1 ci-dessous.

\section{Analyses des paramètres physiques}

Les valeurs des paramètres physiques montrent que la température des eaux souterraines du bassin de Timia, varie de 23 à $32{ }^{\circ} \mathrm{C}$, avec une moyenne de $27^{\circ} \mathrm{C}$ et un écart type de $1,5^{\circ} \mathrm{C}$. Les valeurs du $\mathrm{pH}$ mesurées dans la nappe alluviale varient de 6,4 à 8,2, avec une moyenne de 7,3 et un écart type de 0,5. La conductivité électrique des eaux du bassin de Timia varie de $107 \mu \mathrm{s} / \mathrm{cm}$ à $5480 \mu \mathrm{s} / \mathrm{cm}$, avec une moyenne de $920 \mu \mathrm{s} / \mathrm{cm}$, et un écart type de $1277 \mu \mathrm{s} / \mathrm{cm}$. La conductivité électrique la plus élevée $(5480 \mu \mathrm{s} / \mathrm{cm})$ a été observée dans la source de Tassinik .

Il est à noter que quatre (4) sources carbo-gazeuses (socle fissuré) présentent des conductivités particulières avec des valeurs supérieures à trois mille (3000) $\mu \mathrm{s} / \mathrm{cm}$. Il s'agit des sources de Tassinik, Tatâ̂, Tighraya, Téghazer, dont les conductivités sont respectivement: $5480 \mu \mathrm{s} / \mathrm{cm}, 4598 \mu \mathrm{s} / \mathrm{cm}, 3979 \mu \mathrm{s} / \mathrm{cm}$, 
$3725 \mu \mathrm{s} / \mathrm{cm}$. Par ailleurs, certains points captant les réservoirs d'altérites ont des conductivités électriques très élevées.

\section{Analyse des ions majeurs}

L'ordre d'abondance des cations pour l'ensemble des eaux souterraines du bassin de Timia est de : $\mathrm{Na}^{+}>\mathrm{Mg}^{2+}>\mathrm{Ca}^{2+}>\mathrm{K}^{+}$. Néanmoins, dans les eaux de la nappe alluviale, il arrive que le calcium prédomine donnant la formule ionique suivante : $\mathrm{Ca}^{2+}>\mathrm{Na}^{+}>\mathrm{Mg}^{2+}>\mathrm{K}^{+}$. Le potassium est le cation le moins abondant dans les eaux souterraines du bassin de Timia.

Concernant les anions, l'ordre d'abondance est le suivant : $\mathrm{HCO}_{3}{ }^{-}>\mathrm{Cl}^{-}>\mathrm{SO}_{4}{ }^{2-}$ $>\mathrm{NO}_{3}{ }^{-}>\mathrm{F}^{-}$. Le bicarbonate est l'ion dominant, il représente $83 \%$ de la charge anionique totale. Le bicarbonate est particulièrement très élevé dans les eaux des sources et des altérites.

\section{Faciès hydrochimiques}

Les eaux souterraines du bassin de Timia (Figure 3), montrent que les faciès chimiques sont principalement de type bicarbonaté calcique (52\%); bicarbonaté sodique et potassique $(42 \%)$ dont $13 \%$ sont de type hyper bicarbonaté sodique. Les faciès chloruré et sulfaté calcique $(4 \%)$ et chloruré sodique et potassique ou sulfaté sodique $(2 \%)$ sont faiblement représentés. Le chlorure, le sulfate, et le potassium présentent souvent une teneur faible, sauf pour quelques points spécifiques.

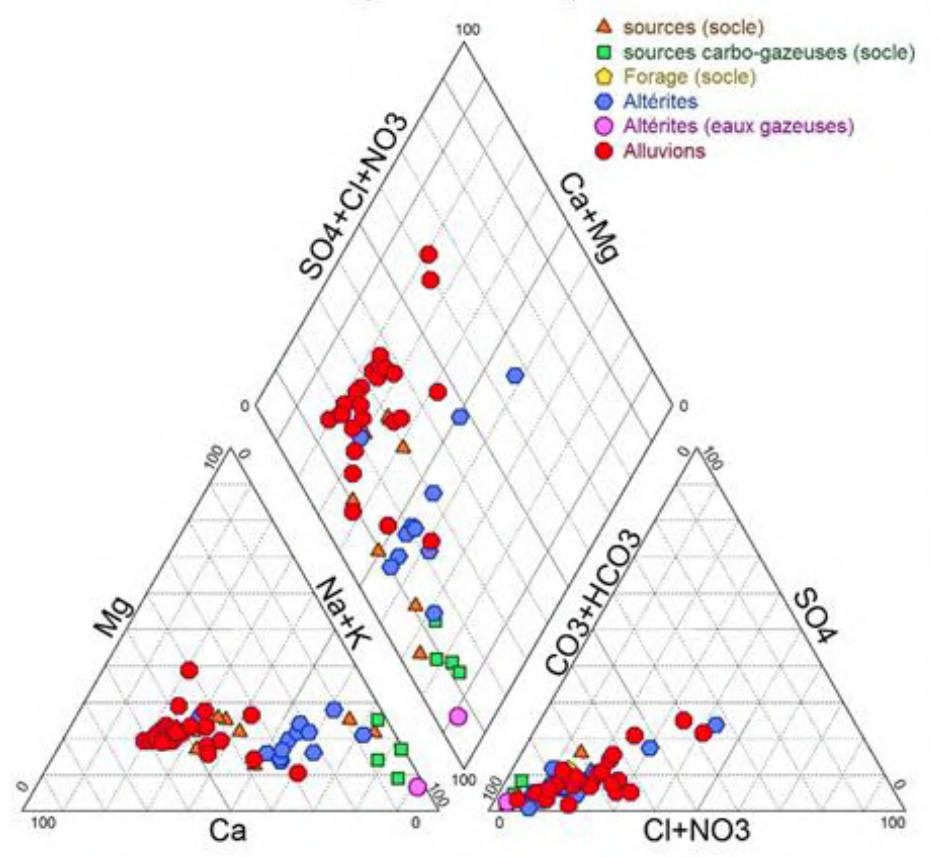

Figure 3: Diagramme de Piper des eaux souterraines du bassin de Timia. 
Tableau 1 : Résultats des analyses chimiques des eaux échantillonnées en meq/l. Conductivité en $\mu \mathrm{S} / \mathrm{cm}$; $\mathrm{T}$ en ${ }^{\circ} \mathrm{C}$

\begin{tabular}{|c|c|c|c|c|c|c|c|c|c|c|c|c|c|c|c|}
\hline Nom & longitude & latitude & $\mathbf{T ~ C ~}^{\circ}$ & pH & $\mathrm{CE} \mu \mathrm{s} / \mathrm{cm}$ & $\mathrm{Ca}++$ & Mg & $\mathrm{Na}+$ & $\mathbf{K}+$ & HCO3- & F- & Cl- & SO4- & NO3- & Balance \\
\hline AMERIGUE_CENTRE & 8,78263 & 18,10073 & 28,60 & 7,50 & 470,00 & 1,40 & 0,67 & 2,48 & 0,03 & 3,14 & 0,09 & 0,59 & 0,48 & 0,31 & $-0,40$ \\
\hline AMER_2_MUSTAPHA & 8,78264 & 18,10064 & 29,10 & 7,53 & 715,00 & 2,25 & 1,08 & 3,35 & 0,08 & 3,51 & 0,09 & 1,66 & 1,17 & 0,34 & $-0,05$ \\
\hline AMERIGUE_DJIBRIL & 8,78387 & 18,0965 & 28,88 & 7,76 & 530,00 & 1,60 & 0,92 & 2,91 & 0,03 & 4,43 & 0,12 & 0,34 & 0,40 & 0,27 & $-0,97$ \\
\hline PUIT_MINTINA & 8,74655 & 18,31085 & 27,00 & 7,29 & 403,00 & 1,75 & 0,92 & 1,22 & 0,08 & 3,31 & 0,09 & 0,25 & 0,19 & 0,10 & 0,30 \\
\hline PUIT_COGERAT & 8,76468 & 18,13966 & 25,00 & 7,17 & 141,00 & 0,65 & 0,25 & 0,39 & 0,10 & 0,87 & 0,03 & 0,17 & 0,13 & 0,10 & 3,83 \\
\hline TANOUT & 8,76468 & 18,13965 & 24,70 & 7,11 & 184,00 & 0,95 & 0,42 & 0,30 & 0,10 & 1,30 & 0,02 & 0,28 & 0,13 & 0,10 & $-1,54$ \\
\hline KIRIP-KIRIP_VILLAGE & 8,65985 & 18,0839 & 29,50 & 7,68 & 852,00 & 1,95 & 1,83 & 4,61 & 0,05 & 6,88 & 0,04 & 0,68 & 1,02 & 0,16 & $-1,94$ \\
\hline KIRIP_ECOLE & 8,67772 & 18,05993 & 27,10 & 7,77 & 1671,00 & 1,85 & 4,58 & 9,83 & 0,13 & 13,46 & 0,06 & 2,42 & 0,81 & 0,90 & $-3,74$ \\
\hline ABARAK_TAMAT & 8,67694 & 18,06066 & 27,30 & 8,38 & 946,00 & 0,80 & 2,17 & 7,17 & 0,18 & 8,07 & 0,08 & 0,73 & 0,27 & 0,34 & 4,17 \\
\hline ABARAK_ABDA & 8,69491 & 18,0612 & 26,20 & 8,19 & 4017,00 & 0,95 & 3,33 & 44,70 & 0,49 & 42,98 & 0,09 & 1,44 & 1,13 & 0,08 & 3,95 \\
\hline AYASTINE & 8,74171 & 18,07292 & 26,70 & 7,71 & 990,00 & 2,00 & 2,25 & 4,78 & 0,31 & 9,63 & 0,03 & 0,96 & 0,10 & 0,03 & $-7,05$ \\
\hline TALEGHATE & 8,73237 & 18,02486 & 29,80 & 7,73 & 503,00 & 2,05 & 1,33 & 1,43 & 0,03 & 4,36 & 0,03 & 0,34 & 0,31 & 0,27 & $-4,68$ \\
\hline TEKAR_SOUGHDOU & 8,77359 & 19,10129 & 26,70 & 7,26 & 259,00 & 1,40 & 0,50 & 0,61 & 0,08 & 1,87 & 0,06 & 0,25 & 0,04 & 0,18 & 3,66 \\
\hline TEKAR_TCHAD & 8,77735 & 18,10338 & 27,70 & 7,20 & 233,00 & 1,30 & 0,50 & 0,48 & 0,08 & 1,42 & 0,05 & 0,25 & 0,23 & 0,21 & 4,22 \\
\hline INFASSASSAN & 8,77812 & 18,11096 & 27,70 & 7,18 & 215,00 & 1,20 & 0,42 & 0,48 & 0,08 & 1,31 & 0,05 & 0,23 & 0,10 & 0,32 & 3,87 \\
\hline GIG_LIMA & 8,77067 & 18,13372 & 25,80 & 7,32 & 181,00 & 1,05 & 0,42 & 0,35 & 0,08 & 1,26 & 0,03 & 0,20 & 0,15 & 0,10 & 4,35 \\
\hline TAM_TAMECHITE & 8,65981 & 18,08392 & 29,90 & 7,77 & 307,00 & 1,85 & 0,58 & 0,57 & 0,03 & 2,64 & 0,01 & 0,23 & 0,10 & 0,16 & $-1,94$ \\
\hline TIMIA_HOUDOU & 8,77706 & 18,11419 & 25,90 & 7,93 & 1775,00 & 3,15 & 3,33 & 8,74 & 0,21 & 5,94 & 0,15 & 4,85 & 4,17 & 2,66 & $-7,02$ \\
\hline TIMIA_ANMINAK & 8,77812 & 18,11375 & 27,60 & 8,02 & 801,00 & 1,70 & 1,25 & 4,70 & 0,08 & 5,76 & 0,04 & 0,87 & 0,50 & 0,21 & 2,25 \\
\hline TIMIA_TASSIGHLYE & 8,78 & 18,11318 & 26,00 & 7,04 & 228,00 & 1,35 & 0,50 & 0,43 & 0,08 & 1,77 & 0,04 & 0,23 & 0,15 & 0,05 & 2,88 \\
\hline TIMIA_TAMIZGUIDA & 8,7836 & 18,11668 & 26,30 & 6,98 & 293,00 & 1,80 & 0,58 & 0,57 & 0,08 & 2,16 & 0,04 & 0,31 & 0,23 & 0,05 & 4,13 \\
\hline TIMIA_CSI & 8,77892 & 18,11215 & 26,70 & 7,20 & 242,00 & 1,45 & 0,50 & 0,48 & 0,08 & 1,92 & 0,04 & 0,25 & 0,17 & 0,05 & 1,65 \\
\hline SRCE_TCHIT_WAZGAR & 8,74586 & 18,0738 & 23,40 & 7,46 & 1738,00 & 1,45 & 4,00 & 10,13 & 0,41 & 16,08 & 0,06 & 1,32 & 0,58 & 0,03 & $-6,13$ \\
\hline SRCE_AKAKARA & 8,75251 & 17,96743 & 20,30 & 7,65 & 435,00 & 1,75 & 0,58 & 2,26 & 0,03 & 3,91 & 0,06 & 0,23 & 0,15 & 0,03 & 2,81 \\
\hline SRCE_TEKARKAR & 8,76662 & 18,09852 & 26,70 & 7,55 & 290,00 & 1,45 & 0,67 & 0,78 & 0,13 & 2,14 & 0,05 & 0,34 & 0,17 & 0,11 & 3,71 \\
\hline SRCE_TIGHRAYA & 8,75368 & 18,07815 & 29,00 & 8,31 & 3979,00 & 0,25 & 8,17 & 37,78 & 1,87 & 42,28 & 0,05 & 4,14 & 1,50 & 0,03 & $\mathbf{0 , 0 7}$ \\
\hline SRCE_TOZAYATE & 8,75377 & 18,07812 & 25,40 & 7,30 & 465,00 & 1,90 & 1,25 & 1,65 & 0,15 & 4,40 & 0,05 & 0,34 & 0,13 & 0,03 & 0,14 \\
\hline SRCE_TIFADAKENE & 8,79567 & 18,21196 & 21,10 & 7,78 & 586,00 & 1,95 & 1,25 & 1,61 & 0,05 & 3,91 & 0,04 & 0,93 & 0,63 & 0,13 & $-7,40$ \\
\hline SRCE_TAGANJIR & 8,76492 & 17,98385 & 29,50 & 7,60 & 3081,00 & 1,65 & 7,58 & 24,52 & 1,33 & 35,26 & 0,04 & 1,30 & 0,60 & 0,06 & $-3,01$ \\
\hline SRCE_TEGHAZER & 8,73244 & 18,02483 & 27,70 & 7,27 & 3725,00 & 0,80 & 10,00 & 27,13 & 1,67 & 38,04 & 0,05 & 3,97 & 1,90 & 0,03 & $-5,26$ \\
\hline SRCE_TASSINIK & 8,76686 & 18,51207 & 28,50 & 6,87 & 5480,00 & 3,20 & 5,50 & 49,09 & 2,90 & 61,44 & 0,09 & 2,70 & 5,85 & 0,03 & $-7,22$ \\
\hline SRCE_TATAI & 8,73257 & 18,30542 & 25,10 & 6,93 & 4598,00 & 4,05 & 7,42 & 39,78 & 1,31 & 52,47 & 0,05 & 2,11 & 2,69 & 0,03 & $-4,36$ \\
\hline TEGUERE_ECOLE & 8,81521 & 18,19005 & 25,40 & 7,32 & 456,00 & 2,00 & 1,17 & 1,30 & 0,03 & 3,55 & 0,05 & 0,48 & 0,27 & 0,18 & $-0,30$ \\
\hline
\end{tabular}




\section{Origines et processus de minéralisation des eaux souterraines}

Pour les eaux souterraines du bassin de Timia, il en ressort que sur l'axe $\mathrm{F} 1(50 \%)$ se regroupent la conductivité électrique (CE), les ions $\mathrm{Mg}^{2+}$, $\mathrm{Na}^{+}, \mathrm{K}^{+}, \mathrm{HCO}_{3}{ }^{-}, \mathrm{Cl}^{-}, \mathrm{SO}_{4}{ }^{2-}$ et la $\mathrm{pCO}_{2}$ (Figure 4 a). L'association de ces ions autours de l'axe F1 montre que celui-ci traduit la principale source de minéralisation des eaux des aquifères et révèle également l'interaction d'autres sources secondaire de la minéralisation des eaux. En observant les principales sources de la minéralisation des eaux (Figure 4b), sur l'axe F1 (deux cercles vert), deux sous-groupes se distinguent : le premier composé par le $\mathrm{Mg}^{2+}, \mathrm{Na}^{+}, \mathrm{K}^{+}, \mathrm{HCO}_{3}{ }^{-}, \mathrm{pCO}_{2}$ et le second est représenté par les ions $\mathrm{Cl}^{-}$et $\mathrm{SO}_{4}{ }^{2-}$. Le premier sous-groupe traduit la minéralisation naturelle des eaux et/ou l'intensité de l'altération des roches. Leur regroupement indique également qu'ils proviennent d'une même source, l'inter action eaux-roche. Le deuxième sous-groupe, formé par les ions $\mathrm{Cl}^{-}$et $\mathrm{SO}_{4}{ }^{2-}$ exprime un apport anthropique donc ils proviennent d'une source superficielle. Le facteur F2 (15\%), regroupant le $\mathrm{NO}_{3}^{-}$et le $\mathrm{F}^{-}$, exprime l'influence des activités anthropiques dans la minéralisation des eaux, traduite particulièrement par la présence du $\mathrm{NO}_{3}{ }^{-}$. Le facteur $\mathrm{F} 3$ représentant seulement $11 \%$ de la variance totale, oppose le $\mathrm{pH}$ et le $\mathrm{Ca}^{2+}$. Cette corrélation négative serait due à la dissolution des minéraux riches en ions $\mathrm{Ca}^{2+}$ dans un milieu faiblement acide.
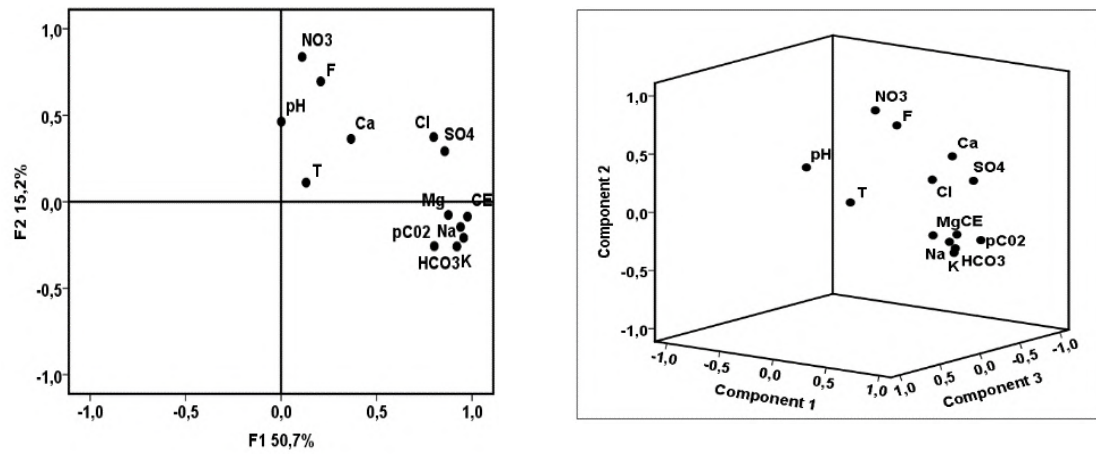

Figure 4: a) Projection des variables sur les plans F1 et F2,b) et F1.F2.F3

La projection des échantillons dans l'espace réduit de deux composantes principales F1 et F2 (Figure 5), montre que les eaux se regroupent en fonction de leur degré de minéralisation et/ou de leur pollution. Les eaux influencées par les facteurs internes (minéralisation naturelle) se situent sur l'axe F1, par contre les eaux polluées, c'est à dire influencées par les apports externes (apports anthropiques), se situent plut tôt dans le plan F2 (opposé au plan F1). 


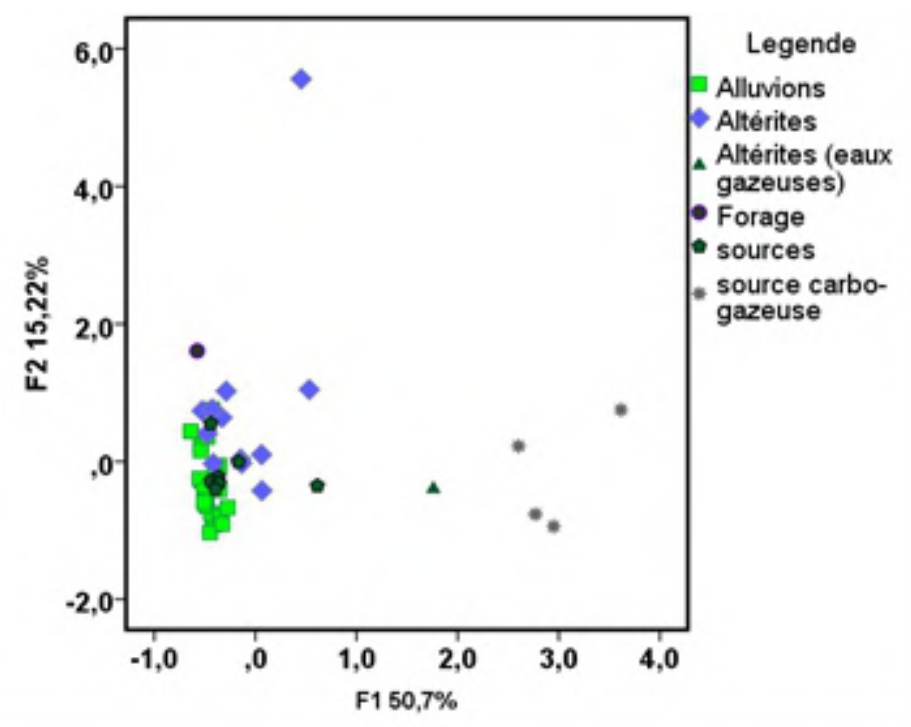

Figure 5: Analyse de l'espace des individus dans les plans F1 et F2

\section{Discussion}

Les températures des eaux souterraines augmentent de l'aquifère alluvionnaire (superficiel) aux réservoirs des altérites et de socle fissurés (respectivement de $26^{\circ} \mathrm{C}, 27^{\circ} \mathrm{C}$ et $28^{\circ} \mathrm{C}$ ). La température des eaux de la nappe alluviale est proche de celle de l'air $(26,9)$ et montre que les nappes superficielles sont soumises aux variations saisonnières de la température de l'air (Ousmane, 1988). Par ailleurs, la nappe des altérites semble avoir une température intermédiaire entre celle de la nappe alluviale et du socle profond.

Les fortes valeurs du $\mathrm{pH}$, supérieures à 8 unités $\mathrm{pH}$, mesurées dans les réservoirs d'altérites, du socle, et des alluvions sont basiques et peuvent être en relation avec une fermeture éventuelle de ces systèmes aquifères. Pour les autres eaux, les valeurs de $\mathrm{pH}$ sont légèrement acides à neutres. Ceci semble être en accord avec la nature géologique des formations aquifères généralement acides (sables) pour la nappe alluviale et (granites altéré et ou fissuré) pour les altérites et le socle.

Les valeurs des conductivités sont plus élevées dans les nappes du socle (sources) et celles des altérites que dans la nappe alluviale traduisant un renouvellement rapide des eaux de la nappe alluviale par rapport aux autres systèmes.

Les eaux de la nappe du socle montrent principalement deux faciès chimiques : l'un de type bicarbonaté calcique et magnésien (54\%) et l'autre de type bicarbonaté sodique (42\%) (fig.58) comme la majorité des eaux souterraines en zone de socle de l'Afrique de l'ouest (Ousmane, 1988 ; Joseph et al., 1992 ; Adiaffi et al., 2009 ; Lasm et al., 2011 ; Babaye,2012 ; Babaye et al.,2016; Illias,2018). 
Il est à noter que quatre sources qui présentent un faciès hyper bicarbonaté sodiques drainant des accidents majeurs sont carbo-gazeuses. Ces fortes concentrations en bicarbonates se rencontrent généralement en régions des fractures en distensions ( Sarro et al., 1982 ; Joseph et al.,1988).

En zone de socle, l'hydrolyse des minéraux silicatés est le principal phénomène dans l'acquisition de la minéralisation des eaux souterraines. Ainsi, un temps de séjour prolongé entre l'eau est la roche entraînent une production des ions $\mathrm{Ca}^{2+}, \mathrm{Na}^{2+}, \mathrm{Mg}^{2+}, \mathrm{K}^{+}$et en ions bicarbonates. Ces derniers sont prépondérants dans les eaux souterraines de socle en Afrique de l'ouest et en accord avec plusieurs travaux (Lasm et al., 2011; Abdou Babaye, 2012 ; Babaye et al.,2016 ; Illias, 2018).Toutefois, ces eaux sont caractérisées par une faible teneur en ion potassium s'expliquant par l'adsorption de celui-ci par les argiles de néoformation et les végétaux (Oga et al.,2009).

La minéralisation des eaux reflète la nature de la roche encaissante (Adiaffi et al., 2009). Pour la nappe alluviale (Figure 6), les ions $\mathrm{Ca}^{2+}$ et $\mathrm{Mg}^{2+}$ (alcalino-terreux) sont moyennement liés à la minéralisation totale $(\mathrm{r}=0,47)$. Pour les alcalins $\left(\mathrm{K}^{+}\right.$et $\left.\mathrm{Na}^{+}\right)$, l'ion $\mathrm{K}^{+}$ne montre aucune relation significative avec celle-ci tandis que le $\mathrm{Na}^{+}$révèle une forte corrélation $(\mathrm{r}=0,88)$ avec cette dernière. Ces résultats montrent que le $\mathrm{Na}^{+}$est l'ion dominant dans le processus de minéralisation des eaux de la nappe alluviale, suivi par les ions $\mathrm{Ca}^{2+}$ et $\mathrm{Mg}^{2+}$ (Joseph, 1990).

Dans les eaux d'altérites (Figure 6), les alcalino-terreux $\left(\mathrm{Mg}^{2+}\right.$ et $\left.\mathrm{Ca}^{2+}\right)$, sont faiblement à très faiblement liés à la minéralisation totale avec des indices de corrélation respectifs de 0,42 et 0,09. Quant aux alcalins $\left(\mathrm{Na}^{+}\right.$et $\left.\mathrm{K}^{+}\right)$, l'ion $\mathrm{Na}^{+}$est fortement lié à la minéralisation totale $(\mathrm{r}=0,97)$ par contre le $\mathrm{K}^{+}$ montre une corrélation moyenne $(\mathrm{r}=0,66)$. Ainsi, il apparaît à partir de ces indices de corrélations que les ions $\mathrm{Na}^{+}$et $\mathrm{K}^{+}$jouent un rôle prédominant dans la minéralisation des eaux des altérites. La dominance des alcalins dans les eaux des alluvions et des altérites pourrait s'expliquer par la nature acide (alluvions issus de l'altération du granite, granite altéré) de leur encaissant (Babaye et al., 2016, Illias,2018) .

Pour les eaux des sources (socle) (Figure 6), le $\mathrm{Mg}^{2+}$ est assez bien lié à la minéralisation totale $(\mathrm{r}=0,77)$. Le $\mathrm{Ca}^{2+}$ ne montre aucune corrélation avec la minéralisation totale. Le $\mathrm{Na}^{+}$et le $\mathrm{K}^{+}$sont très fortement liés à la salinité, respectivement $r=0,99$ et 0,92 . Ainsi les ions $\mathrm{Na}^{+}, \mathrm{K}^{+}$et $\mathrm{Mg}^{2+}$ sont responsables de la minéralisation des eaux du socle. En plus, ces ions moins importants dans les eaux des altérites que celles du socle montrant le rôle prépondérant de l'hydrolyse dans ces dernières. De plus, la présence des

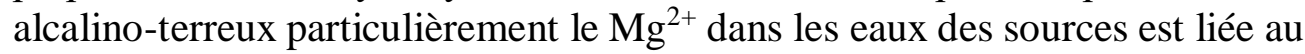
fait certaines de ces sources ont un encaissant basique (basalte). 
S'agissant des bicarbonates (Figure 7), la très forte corrélation entre l'ion $\mathrm{HCO}_{3}{ }^{-}$avec la minéralisation (pour tous les aquifères) prouvent que cet ion serait issu de la dissolution des minéraux silicatés.

Pour les autres anions $\left(\mathrm{SO}_{4}{ }^{2-}, \mathrm{Cl}^{-}, \mathrm{NO}^{-}\right)$, aucune relation entre ceuxci et la minéralisation totale dans les eaux de la nappe alluviale et celles des altérites n'est observée. Par contre, dans les eaux des sources (socle), une assez bonne corrélation s'observe respectivement entre les ions $\mathrm{SO}_{4}{ }^{2-}$ et la salinité $(\mathrm{r}=0,69)$ et cette dernière et les ions $\mathrm{Cl}^{-}(\mathrm{r}=0,67)$. La présence de ces ions dans les eaux du socle s'expliquerait par les activités volcaniques dans l'Aïr, susceptibles de dégager du chlore et des sulfures (Joseph,al.,1991).

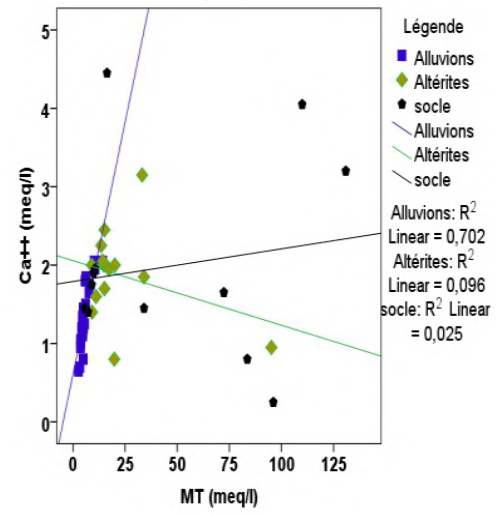

a) relation entre $\mathrm{Ca}^{2+}$ et $\mathrm{MT}$

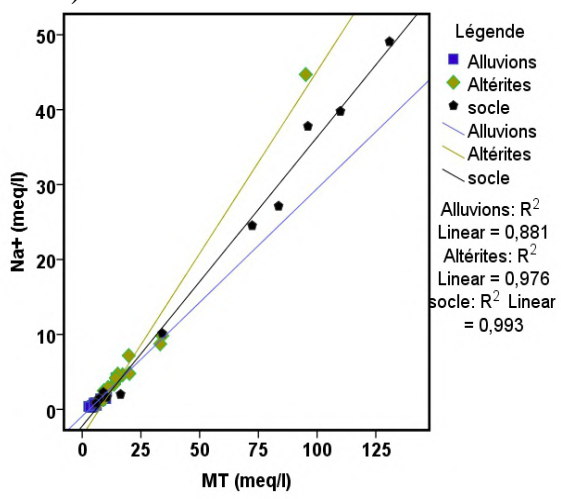

c) relation entre $\mathrm{Na}+$ et $\mathrm{MT}$

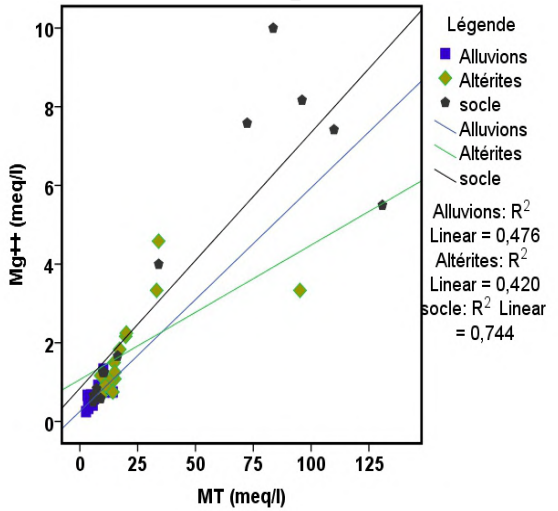

b) relation entre $\mathrm{Mg}^{2+}$ et $\mathrm{MT}$

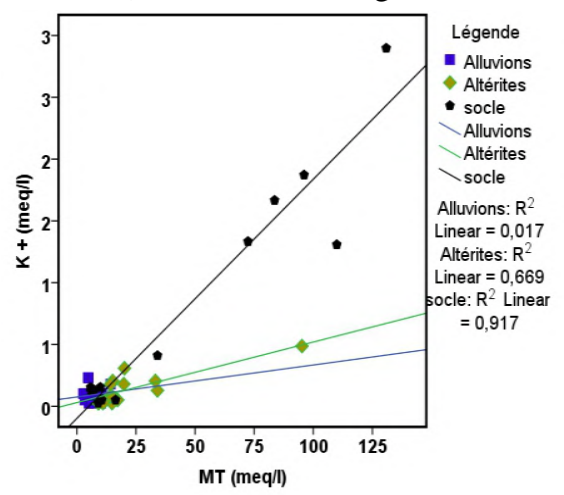

d) relation entre $\mathrm{K}+$ et $\mathrm{MT}$

Figure 6: Relation entre la minéralisation totale et les cations 


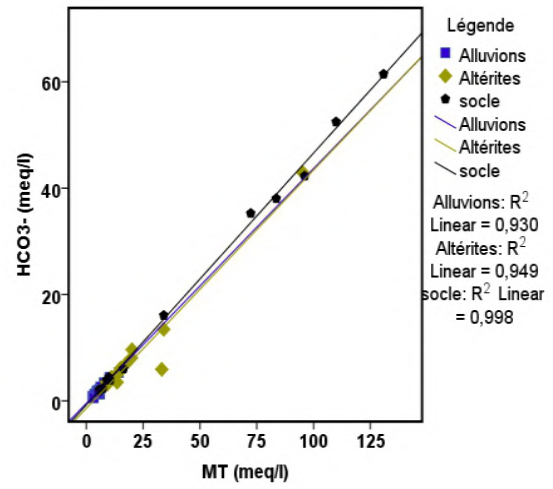

a) relation entre $\mathrm{HCO}_{3}^{-}$et $\mathrm{MT}$

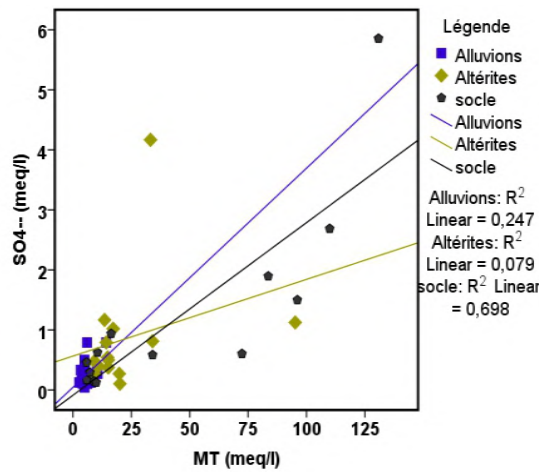

c) relation entre $\mathrm{SO} 4$ - et $\mathrm{MT}$

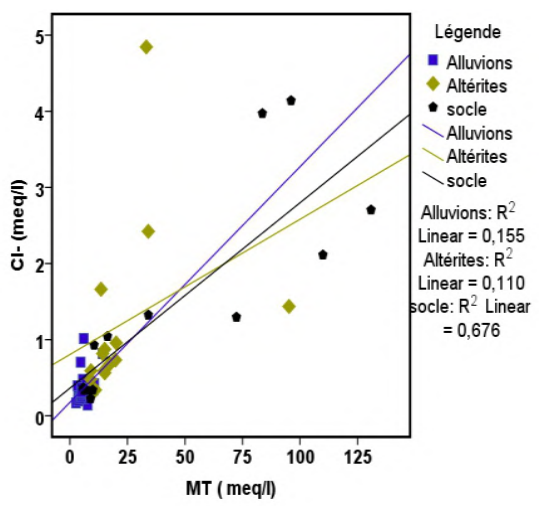

b) relation entre $\mathrm{Cl}^{-}$et $\mathrm{MT}$

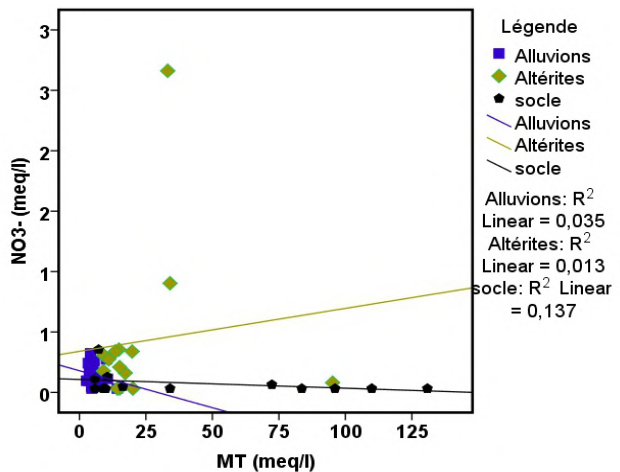

d) relation entre $\mathrm{NO} 3$ - et $\mathrm{MT}$

Figure 7: Relation entre la minéralisation totale et les anions

\section{Conclusion}

L'étude hydrogéochimique des eaux souterraines a permis de distinguer les eaux de la nappe alluviale légèrement acide à neutre des aquifères des altérites et de socle avec des valeurs de $\mathrm{pH}$ légèrement basique. Ces eaux sont faiblement minéralisées dans leurs grandes majorités. Toutefois, les eaux à forte minéralisation se rencontrent dans la nappe de socle particulièrement dans les eaux des sources drainant les fractures majeures, et certains ouvrages captant la nappe des altérites. Les facies chimiques des eaux souterraines sont dominés par les facies de type bicarbonatés calciques et magnésiens représentant $52 \%$ des échantillons analysés. Les facies bicarbonatés sodiques et potassiques sont aussi important (29\%) tandis que les eaux hyper-bicarbonatées sodiques (13\%) sont faiblement représentées. Les facies de type chloruré et sulfaté calcique (4\%) et chloruré sodique et potassique ou sulfaté sodique (2\%) sont minoritaires suggérant des apports des surfaces. Les sources de la minéralisation des eaux sont principalement de trois types: les apports superficiels (pollution), l'hydrolyse des minéraux silicatés et les activités volcaniques. 


\section{References:}

1. Abdou Babaye MS. (2012). Evaluation des ressources en eau souterraine dans le bassin de Dargol (Liptako Niger). PhD Thesis, Université Liège, p235.

2. Abdou Babaye MS. ; Philpe Orban ;Boureima Ousmane ;Guillaume Favreau ;Serge Brouillere ;Alain Dassargue. (2016). Characterization of recharge mechanisms in precambrian basement aquifer in semi-arid south-west Niger. Hydrology journal 10.107/s10040-018-1799-xx.

3. Adiaffi B. (2008). Adiaffi B. 2008. Apport de la géochimie isotopique, de l'hydrochimie et de la télédétection à la connaissance des aquifères de la zone de contact "socle bassin sédimentaire" du Sud-Est de la Côte d'Ivoire. Thèse de doctorat, Université Paris Sud, p.230.

4. Adiaffi B, Marlin C, Oga YMS, Massault M, Noret A, Biémi J. (2009). Palaeoclimatic and deforestation effect on the coastal fresh groundwater resources of SE Ivory Coast from isotopic and chemical evidence. J. Hydrol., 1-2: 130-141.

5. Aranyossy J. F., Joseph A. (1985). Etude géochimique et isotopique des aquifères de l'Aïr (Niger). A.I.E.A., TA-2330,43 p.

6. Armand C., Dehays H. (1987). Reconnaissance géophysique par prospection électrique dans six vallées de l'Aïr. B.R.G.M.Ministère de l'Hydraulique et de l'Environnement du Niger. pp 5-37.

7. Black R., Jaujou M., Pellaton C. (1967). Notice explicative sur la carte géologique de l'Air au 1/ 500000 . Ed. du BRGM, Paris, $57 \mathrm{p}$

8. BLack R. (1978). Propos sur le Panafricain. Bull. Soc. Geol. Fr., 20, pp. 843-850.

9. El Hamet M.O. (1983). Analyse géologique et pétrographique de la formation de Tarat dans les carrières SOMAÏR (Paléozoïque supérieur). Essai d'interprétation paléoclimatique à la lumière de l'épisode glaciaire dévono-carbonifère. Thèse spécialité Université de Dijon et Niamey, 279 pages.

10. Garnier J.M., Fontes J.Ch. (1980). Reconnaissance hydrochimique et isotopique des eaux souterraines de la cuvette d'Agadez (Niger). Rapport O.R.S.T.O.M.

11. Greigert J., Pougnet R. (1967). Essai de description des formations géologiques de la république du Niger. Ministère des Travaux Publics, des Transports, des Mines et de l'Urbanisme. Direction mines et géologie. Publication $n^{\circ} 3$ Edit. du B.R.G.M, 273

12. Illias A. (2018). Évaluation des ressources en eaux souterraines du bassin de Timia (massif de l'Ä̈r, Nord du Niger) : impacts de la 
variabilité climatique et des activités anthropiques. Thèse de doctorat université Abdou Moumouni de Niamey, 198 Pages

13. Joseph A. (1990). Recharges et paléorecharges des nappes en région subdésertiques au Niger. Thèse de l'Université Paris VI, $163 \mathrm{p}$.

14. Joseph A., Aranyossy J. F. (1987). Etude géochimique et isotopique des aquifères de la plaine de 1'lrhazer. I.A.E.A. NER/8/003. Report of an expert mission. Division of technical assistance and coopération. $33 \mathrm{p}$.

15. Joseph A., Frangi J.P. (1989). Modélisation d'une recharge effective de nappe alluviale en région subdésertique: cas de l'oasis du Téloua, Niger. Journal of African Earth Sciences, vol. 9 no 2 pp 317-326.

16. Joseph. A (1992): L'Aïr « château d'eau » de la bande désertique du Ténéré (Niger). Revue de géographie alpine.1991, Tome 79 N¹. PP 71-86.

17. Karche J.P. (1979). Remarques morphologiques et structurales sur le massif volcanique du Todgha (sud de l'Aïr). Ann. Univ. Niamey; tome II, pp. 97-108.

18. Karche J.P., Vachette M. (1978). Age et migration de l'activité magmatique dans les complexes paléozoïques du Niger, conséquences. Bull. Soc. géol. France. (7) t. XX no 6, pp. 941953.

19. Lasm T, De Lasme O, Oga MSY, Youan Ta M, Baka D, Kouame F, Yao TK. (2011). Caractérisation hydrochimique des aquifères fissurés de la région de San Pedro (Sud-Ouest de la Côte d'Ivoire). Int. J. Biol. Chem. Sci., 5(2): 642-662.

20. Lasm L, Jourda JP. (2011). Étude hydrogéochimique des eaux des aquifères de fractures du socle Paléoprotérozoïque du Nord-Est de la Côte d'Ivoire : Cas de la région de Bondoukou. Afriq. Sci., 08(3) : $51-68$.

21. Moreau C. (1982). Les complexes annulaires anarogéniques à suites anorthositiques de l'Aïr central et septentrional (Niger). Thèse d'état, Nancy, $356 \mathrm{p}$.

22. Morel A. (1985). L'apport des glacis anciens de l'Air (Niger) à la connaissance des variations climatiques du pléistocène inférieur d'Afrique occidentale. "Changements globaux en Afrique durant le Quaternaire, passé, présent et futur”. INQUA-ASEQUA Symposium international Dakar, Ed. de l'ORSTOM, Coll. Trav. et Dot. no 197, pp. 321-325.

23. Oga MS, Lasm T, Yao TK, Soro N, Saley MB, Kouassi D, Gnamba F. (2009). Caractérisation chimique des eaux des aquifères 
de fractures: Cas de la Région de Tiassalé en Côte D'Ivoire. European Journal of Scientific Research, 31(1):72-87.

24. Ousmane B. (1988). Etude géochimique et isotopique des aquifères du socle de la bande sahélienne du Niger (Liptako, Sud Maradi et Zinder Est). Thèse de doctorat d'état, Université de Niamey. 175 pages

25. Piper A. M. (1944). A graphic procedure in geochemical interpretation of water analyses.

26. Raulais M. (1959). Esquisse géologique sur le massif cristallin de l'Aïr (Niger). Bull. Soc. géol. Fr., 7e série, t. 1, no 2, pp. 207223.

27. Roussin-Bariac M. (1985). Etude hydrochimique et isotopique d'une nappe alluviale sous climat semi-aride (cuvette d'Agadez, Niger). Thèse Université Paris-Sud, 218 p.

28. Schulz E. (1987). Die Holozàne Vegetation der zentralen Sahara (N-Mali, N-Niger, SW Libyen). Palaeoecology of Africa, 18, 143161

29. Sempere T. (1981). Le contexte sédimentaire du gisement d'uranium d'Arlit (République du Niger). Mémoire Thèse, Ecole Nationale Supérieure des Mines de Paris, 374 pages

30. Valsardieu C. (1971). Cadre géologique et paléogéographique des minéralisations de charbon, de cuivre et d'uranium de la région d'Agadès (République du Niger). Thèse Doctorat. Etat, Nice, 518 p.

31. Yahaya M. (1992). Dynamique sédimentaire du Guézouman et des formations viséennes sous-jacentes en liaison avec la tectonique, le volcanisme et le climat. Mémoire de Thèse Université de Bourgogne, 355 pages. 